An. MED. InTERNA (Madrid) Vol. 19, N. $^{\circ} 9$, pp. $473-476,2002$

\title{
Infecciones por Arcanobacterium haemolyticum: un patógeno emergente
}

\author{
J. L. PUERTO ALONSO, P. GARCÍA-MARTOS, J. A. GIRÓN GONZÁLEZ* \\ Servicios de Microbiología y *Medicina Interna. Hospital Universitario Puerta del Mar. \\ Cádiz
}

\begin{abstract}
RESUMEN
Arcanobacterium haemolyticum es un bacilo grampositivo perteneciente, hasta hace poco, al género corynebacterium, y clasificado recientemente en un nuevo género, con una sola especie. Su principal reservorio es el hombre. Ha sido aislado de la piel y faringe de individuos sanos, pero también como causa de infección, principalmente faringitis, en niños, y úlceras cutáneas crónicas, en diabéticos. Con menos frecuencia puede dar lugar a osteomielitis, meningitis, abscesos, neumonía, endocarditis y sepsis. El diagnóstico se ve dificultado por su doble cualidad de comensal y patógeno. Para el tratamiento de estas infecciones no existen protocolos establecidos, aunque la mayoría de las cepas aisladas son sensibles a penicilina, eritromicina, clindamicina y tetraciclina. Para el tratamiento de las infecciones profundas, se recomienda penicilina a dosis altas con o sin aminoglucósidos.
\end{abstract}

PALABRAS CLAVE: Arcanobacterium haemolyticum. Infecciones. Revisión. Microbiología. Diagnóstico. Tratamiento.

\author{
INFECTIONS BY ARCANOBACTERIUM HAEMOLYTICUM: AN \\ EMERGING PATHOGEN
}

\begin{abstract}
Arcanobacterium haemolyticum is a grampositive rod wich belon ged, untill a short time ago, to Corynebacterium genus, and recently classified in a new genus, with only one specie. Human is the main reser voir. It has been isolated from the skin and pharinx of healthy indivi duals, but also it is cause of infection, specially pharingitis, in children, and chronic cutaneous ulcus, in diabetic patients. Less frequently, it is cause of osteomyelitis, meningitis, pneumonia, abscess, endocarditis and sepsis. Diagnosis is difficult because its double quality: comensal and pathogen. There are not established guidelines for the treatment of these infections, although most of isolated strains are susceptibles to penici llin, erythromicin, clindamycin and tetracycline. High doses of penici llin, with or without gentamicin, it is recommended for the treatment of deep infections.
\end{abstract}

KEY WORDS: Arcanobacterium haemolyticum. Infections. Review. Microbiology. Diagnosis. Treatment.

Puerto Alonso JL, García-Martos P, Girón González JA. Infecciones por Arcanobacterium haemolyticum: un patógeno emergente. An Med Interna (Madrid) 2002; 19: 473-476.

\section{INTRODUCCIÓN}

Arcanobacterium haemolyticum es un bacilo grampositivo, comensal humano en faringe y piel, implicado fundamentalmente en faringitis no estreptocócica, en adolescentes y adultos jóvenes, e infecciones de heridas (1-3). También se describe, aunque excepcionalmente, como causante de infecciones sistémicas y de órganos, principalmente endocarditis, osteomielitis, meningitis y neumonía (4-9). Estas situaciones graves ocurren primordialmente en pacientes con enfermedad de base, como alcoholismo, diabetes o neoplasias. A menudo, la infección se produce en asociación con otros microorganismos, circunstancia que algunos autores aprovechan para argumentar en contra del papel patógeno de A. haemolyticum $(10-12)$
En este trabajo revisamos la situación actual de las infecciones causadas por este microorganismo, en cuanto a su epidemiología, manifestaciones clínicas, tratamiento, y su importancia como patógeno emergente.

\section{MICROBIOLOGÍA}

Arcanobacterium haemolyticum se describió por primera vez en 1946, aislándose en soldados americanos destinados en el Pacífico Sur tras la segunda guerra mundial, encuadrándose, en un principio, dentro del género Corynebacterium (Corynebacterium haemolyticum) (13). Hoy día y desde el año 1982, se ha separado en el nuevo género Arcanobacte rium, con una única especie, relacionado con Actinomyces y

Trabajo aceptado: 28 de enero de 2002

Correspondencia: José Luis Puerto Alonso. Avda. de México. Parque Stadium. Bque. 6, 4-C. 11405 Jerez de la Frontera (Cádiz). 
Corynebacterium, de los que se puede diferenciar por análisis de ácidos grasos y por su capacidad para producir a-manosidasa (14-17).

Se trata de un bacilo grampositivo, ligeramente curvado, con los extremos puntiagudos, aerobio o anaerobio facultativo, inmóvil, que no forma esporas ni produce catalasa, no hidroliza la esculina, ni la urea, ni la gelatina.

Es un microorganismo que crece bien en agar sangre, incubado a $35^{\circ} \mathrm{C}$ durante $48-72$ horas en atmósfera rica en $\mathrm{CO}_{2}$, donde forma, generalmente, colonias pequeñas $(0,1$ $\mathrm{mm}$ a las 24 horas y $0,5 \mathrm{~mm}$ a las 48 horas) rodeadas por una estrecha banda de betahemólisis $(12,18)$. Hay que tener en cuenta que A. haemolyticum hemoliza lentamente, en 48-72 horas, y que la mayoría de los laboratorios resuelven los cultivos de exudados faringeos en 48 horas, con lo que el microorganismo puede pasar desapercibido por no haber producido aún betahemólisis. Sin embargo, esta zona de hemólisis es mayor y se forma más rápidamente si, en lugar de sangre de carnero, empleamos sangre de caballo, de conejo o humana.

A las 48-72 horas los bacilos conservan su morfología típica, pero después de 4 días de incubación, aproximadamente, la observación microscópica de A. haemolyticum puede inducir a error, ya que algunos bacilos se colorean desigualmente, pudiendo confundirse con cadenas cortas de estreptococos. Este hecho, además de la negatividad para la catalasa y la presencia de un halo de betahemólisis, puede llevarnos a errar el diagnóstico.

Atendiendo a la morfología de las colonias, podemos distinguir dos biotipos: liso y rugoso. El biotipo liso es mucho más frecuente, aproximadamente un $75 \%$ de los aislamientos, y se caracteriza por colonias blanquecinas, de perfil bien definido, rodeadas por una zona de moderada a fuerte betahemólisis; el biotipo rugoso es menos frecuente, alrededor del $25 \%$, da lugar a colonias grisáceas, secas, de perfil irregular, y la betahemólisis no se evidencia o es muy débil. Bioquímicamente no existen diferencias entre los dos biotipos, con la excepción de que, alrededor de la mitad de las colonias que pertenecen al biotipo liso fermentan la sacarosa y la trehalosa; además, ninguna de las colonias lisas presenta actividad b-glucoronidasa, mientras que alrededor del $30 \%$ de las rugosas si la producen (19). Por otra parte, parece que hay una relación entre el biotipo y la localización anatómica de la muestra. Así, el biotipo liso se asocia más frecuentemente a infecciones de heridas, mientras que el rugoso se aísla más en muestras del tracto respiratorio superior.

\section{EPIDEMIOLOGÍA Y PATOGENIA}

Aunque A. haemolyticum se puede aislar en animales, el reservorio primario es el hombre, ya que puede encontrarse en piel y faringe de individuos sanos. La dificultad para dignosticar las infecciones por este microorganismo y la falta de referencias en la literatura, explican la inexistencia de datos epidemiológicos concluyentes acerca de las infecciones causadas por el mismo. A. haemolyticum se ha aislado, sobre todo, en muestras de exudados faringoamigdalares y de infecciones de piel, donde produce úlceras crónicas, asociadas con frecuencia a diabetes. Aunque su implicación en faringitis queda recogida en la literatura, se discute su papel como pató- geno en algunos casos, debido fundamentalmente a la imposibilidad de descartar agentes virales y micoplasmas. Se conoce que afecta sobre todo a niños y adultos jóvenes dentro del rango etario entre 10-30 años. En estas edades los estreptococos no son responsables de más del $10-25 \%$ de las faringitis, por lo que se piensa que $A$. haemolyticum debe estar implicado en un porcentaje importante de las mismas, que algunas fuentes sitúan al menos entre el $5-13 \%$ de las atribuidas a estrepto$\operatorname{cocos}(1,2,20)$. Los mecanismos por los que la infección se transmite no son bien conocidos, aunque parece que la transmisión se produce vía aérea por medio de gotitas de saliva. Las infecciones profundas y sepsis son mucho más frecuentes en hombres, pero no se conocen razones que expliquen esta situación (9).

Este microorganismo sintetiza toxinas activas como la betahemolisina, fosfolipasa $\mathrm{D}$ y neuraminidasa, que parecen responsables del daño tisular y del exantema que puede acompañar a la faringitis (20). Así, la inyección subcutánea en cobayas produce necrosis y una amplia zona de edema y eritema. Es posible que estos factores de virulencia también sean responsables de un efecto sinérgico entre A. haemolyticum y otros microorganismos, al facilitar el primero la colonización de los otros, provocando entre ambos un mayor daño tisular (21).

\section{CLÍNICA}

El cuadro clínico de la faringitis, que es agudo, es parecido al producido por Streptococcus pyogenes (grupo A), con una erupción escarlatiniforme inicial (30-70\% de los afectados) y linfadenitis en el $50 \%$ de los pacientes. La severidad de la sintomatología varía desde una ligera irritación de la faringe hasta una inflamación importante con exudado en orofaringe posterior. La erupción, que puede ser el signo inicial y la manifestación predominante, se localiza en tronco y porciones proximales de las extremidades, respetando cara y palmas de las manos, con descamación a medida que progresa la enfermedad. No suele haber signos de afectación sistémica, como fiebre alta o leucocitosis $(1,3)$.

Más raramente $A$. haemolyticum puede ser causante de infecciones profundas y sistémicas. Se han descrito diversos tipos de abscesos causados por este microorganismo: abscesos cerebrales, asociados o no a bacteriemia $(4,7,22)$, un caso de absceso pulmonar (23), otro de partes blandas tras fractura abierta (24), un absceso de mama en una paciente joven, sin puerta de entrada conocida (24), un absceso subperióstico, en tórax, asociado a celulitis orbital, sinusitis y bacteriemia (8), aunque también se ha referido sinusitis y celultis orbital en ausencia de otra focalidad (25), y abscesos tuboovárico, paraamigdalino y paravertebral (26).

También se han referido contados casos de osteomielitis, la mayoría tras fractura y sin acompañarse de bacteriemia $(12,16,27)$, meningitis $(6,9,11,27)$, endocarditis $(5,28,29)$, neumonía $(9,30,31)$ y dos casos de infección articular sin bacteriemia $(24,32)$.

Ocasionalmente, A. haemolyticum puede dar lugar a episodios de sepsis (30). Hasta la fecha, solamente hay descritos 27 casos, tanto en pacientes adultos y de edad avanzada (33-87 años), inmunodeprimidos o con factores de riesgo (diabetes, neoplasia, ADVP), como en pacientes adolescentes o adultos jóvenes inmucocompetentes $(6,8,9,23,25,30)$. 
En los pacientes inmunocomprometidos los focos de infección más frecuentes son la infección de herida sobre pie diabético, neumonía y osteítis metatarsal. En los pacientes inmunocompetentes, los focos de infección más frecuentes son el absceso amigdalino y la sinusitis, aunque el foco infeccioso a veces no logra establecerse. De los 27 casos de sepsis descritos, 11 fueron infecciones mixtas, aislándose junto a A. haemolyticum estreptococos (Streptococcus agalactiae, S. sanguis, S. milleri) y anaerobios (Fusobacterium necrophorum, Prevotella bivia, Bacteroides fragilis, $B$. capillosus) (23). Además, en dos adolescentes existía coinfección por el virus Epstein-Barr (25). Algunos autores han postulado que un descenso de la respuesta inmune en la infección por EBV podría aumentar la invasividad de $A$. haemolyticum. Realmente no conocemos la razón de esta alta tasa de coinfectividad, aunque puede deberse a la síntesis de tóxinas activas por parte de A. haemolyticum, como betahemolisina y fosfolipasa $\mathrm{D}$, que facilitarían la colonización por otros microorganismos.

\section{DIAGNÓSTICO MICROBIOLÓGICO}

El hecho de que A. haemolyticum pueda encontrarse en la faringe y piel de sujetos sanos, dificulta considerablemente el diagnóstico, y la identificación del microorganismo requiere la sospecha por parte del clínico. Los datos suministrados por éste son indispensables para el diagnóstico microbiológico y deberían facilitarse siempre. En nuestra opinión, no deberían valorarse los aislamientos sin sospecha clínica previa.

Arcanobacterium haemolyticum crece bien en agar tripticasa-soja, agar Columbia e infusión de cerebro-corazón, todos con un 5\% de sangre de carnero. Sin embargo, si en lugar de utilizar sangre de carnero usamos sangre de conejo, de caballo o humana, la banda de betahemólisis es más ancha y aparece antes, con lo que mejoramos las condiciones para el diagnóstico $(10,18)$. También debe tenerse en cuenta que A. haemolyti cum crece en medios para estreptococos, lo que puede llevar a confusión con estreptococos con antígenos del grupo Lancefield negativos.

Los criterios microbiológicos para diagnosticar A. hae molyticum se recogen en artículos recientes $(18,21,33)$. El cultivo debe hacerse en agar sangre, mejor si la sangre es de caballo, e incubarlo a $35^{\circ} \mathrm{C}$ en una atmósfera rica en $\mathrm{CO}_{2}$ durante 48-72 horas. Una vez crecido el microorganismo, la identificación debe realizarse siguiendo los criterios descritos por Krech y Hollis (33), que incluyen: betahemólisis en agar sangre, tinción de Gram, producción de catalasa, reducción de nitratos, producción de ureasa, hidrólisis de la gelatina, motilidad, CAMP test, y fermentación de glucosa, maltosa, sacarosa, manitol y xilosa. Estos criterios, sin embargo, no sirven para el biotipado de las cepas, que se realiza por la morfología de las colonias, presencia de betahemólisis, actividad betaglucoronidasa y fermentación de sacarosa y trehalosa (14).

Existen algunos sistemas comerciales útiles para el diagnóstico de bacterias corineformes que son válidos para $A$. hae molyticum $(34,35)$. El más usado es el API Coryne (Biomérieux, France), que consta de 20 pruebas bioquímicas y enzimáticas, que incluyen: catalasa, reducción de nitratos, pirazinaminidasa, pirrolidín-arilamidasa, fosfatasa alcalina, beta-glucoronidasa, beta-galactosidasa, alfa-glucosidasa, Nacetil-beta-glucoaminidasa, ureasa, hidrólisis de esculina, fermentación de glucosa, ribosa, xilosa, manitol, maltosa, lactosa, sacarosa y glucógeno $(35,36)$.

Otros métodos se basan en el análisis de elementos estructurales de la célula. Entre ellos, el más desarrollado es la detección de ácidos grasos, especialmente por cromatografía $(13,17)$.

Trabajos recientes refieren la utilidad de determinados sustratos fluorogénicos (4-metilumbeliferil-a-D-manopi-ranósido) para la identificación de Arcanobacterium y otras bacterias corineformes (37), o la detección de a-manosidasa mediante una prueba de lectura rápida (en cuatro horas), que es útil para diferenciarlo de corinebacterias y Actinomyces pyogenes (14).

\section{TRATAMIENTO}

Según la literatura, A. haemolyticum es sensible a penicilina, aunque estudios más recientes evidencian la existencia de cepas que presentan tolerancia in vitro a este antimicrobiano (38). La mayoría de los estudios también indican que es sensible in vitro a cefalosporinas, vancomicina, aminoglucósidos, clindamicina, ciprofloxacina y macrólidos (eritromicina y azitromicina). Así mismo, parece mostrar bajas CMIs a carbapenemas, rifampicina y teicoplanina (27). Las CMIs para vancomicina son considerablemente más altas que a teicoplanina. Trovafloxacina y ofloxacina parecen activas in vitro, pero trovafloxacina tiene una actividad límite (27). El único antibiótico probado que constantemente se muestra resistente es trimetropim-sulfametoxazol. Otros antimicrobianos a los que en ocasiones puede ser resistente son sulfonamidas y oxacilina $(6,30,39)$

En general, se consigue la curación con penicilina, aunque también se han informado fracasos, incluso en cepas sensibles in vitro, posiblemente debidos a la supervivencia intracelular del microorganismo $(1,12,38,40)$. Sin embargo, estudios in vivo han demostrado que los macrólidos son capaces de erradicar a este microorganismo de la faringe de pacientes afectados, cosa que no consiguen totalmente los betalactámicos $(1,38)$.

Prácticamente no existen recomendaciones para el tratamiento de las infecciones por A. haemolyticum, debido al pequeño número de casos y la poca experiencia acumulada. En el caso de las faringitis, algunos autores recomiendan usar eritromicina, administrada por vía oral, o bien penicilina (3). En cuanto a las infecciones profundas y sepsis, las escasas referencias al respecto sugieren tratar con penicilina a altas dosis, por vía intravenosa, como primera elección, con o sin gentamicina (9). También podrían usarse eritromicina y clindamicina. Por otra parte, todos estos antimicrobianos, excepto los macrólidos, tienen actividad frente a anaerobios, frecuentemente asociados en las infecciones por A. haemolyti $\operatorname{cum}(1,12)$.

En aquellas situaciones donde los betalactámicos no penetren adecuadamente en los tejidos, como en la endocarditis y osteomielitis, es recomendable el empleo de macrólidos o clindamicina junto a rifampicina. En cualquier caso, el tratamiento de las infecciones profundas y sepsis debe elegirse de acuerdo con criterios de susceptibilidad individual y el lugar de asiento de la infección (27). 


\section{Bibliografía}

1. Banck G, Nyman M. Tonsillitis and rash associated with Arcanobacterium haemolyticum. J Infect Dis 1986; 154: 1037-40.

2. Cambier M, Janssens M, Wauters G. Isolation of Arcanobacterium haemolyticum from patients with pharyngitis in Belgium. Acta Clin Belg 1992; 47: 303-7.

3. Gaston DA, Zurowski SM. Arcanobacterium haemolyticum pharyngitis and exanthem. Arch Dermatol 1996; 132: 61-4.

4. Altmann G, Bogokovsky B. Brain abscess due to Arcanobacterium haemolyticum. Lancet 1973; 1: 378-9.

5. Alós LI, Barros C, Gómez-Garcés JL. Endocarditis caused by Arcanobacterium haemolyticum. Eur J Clin Microbiol Infect Dis 1995; 14: 1085-8.

6. Ben-YaacobD, Waron M, Boldur I, Gil I, Sompolinski D. Septicemia due to Arcanobacterium haemolyticum. Isr J Med Sci 1984; 20: 431-3.

7. Chhang WH, Ayyagari A, Sharma BS, Kak VK. Arcanobacterium haemolyticum brain abscess in a child (a case report). Indian J Pathol Microbiol 1991; 34: 145-8.

8. Ford JG, Yeatts RP, Givner LP. Orbital cellulitis, subperiosteal abscess, and septicemia caused by Arcanobacterium haemolyticum: Am J Ophtalmol 1995; 120: 261-2.

9. Skov RL, Sanden VH, Danchell VH, Robertsen K, Ejlertsen T. Systemic and deep-seated infections caused by Arcanobacterium haemolyticum. Eur J Microbiol Infect Dis 1998; 17: 578-82.

10. Funke G, von Graevenitz A, Clarridge JE III, Bernard KA. Clinical microbiology of coryneform bacteria. Clin Microbiol Rev 1997; 10: 125-59.

11. Minarik T, Sufliarsky J, Trupl J, Krcmery VJ. Arcanobacterium haemolyticum invasive infections, including meningitis in cancer patients. J Infect Dis 1997; 34: 91-2.

12. Waagner DC. Arcanobacterium haemolyticum: Biology of the organism and diseases in man. Pediatr Infect Dis J 1991; 10: 933-9.

13. Bernard KA, Bellefeuille M, Ewan EP. Cellular fatty acid composition as an adjunct to the identification of Asporogenous, aerobic gram-positive rods. J Clin Microbiol 1991; 29: 83-9.

14. Carlson P, Kontiainen S. Alpha-mannosidase: a rapid test for identification of Arcanobacterium haemolyticum: J Clin Microbiol 1994; 32: 854-5.

15. Collins MD, Jones D, Schofield GM. Reclassification of "Corynebacte rium haemolyticum" (McLean, Liebow \& Rosenberg) in the genus Arcanobacterium gen. nov. as Arcanobacterium haemolyticum nom. rev., comb. nov. J Gen Microbiol 1982; 128: 1279- 81.

16. Mclean PD, Liebow AA, Roenbreg AA. A haemolytic Corynebacterium resembling Corynebacterium ovis and Corynebacterium pyogenes in man. J Infect Dis 1946; 79: 69-90.

17. Von Graevenitz A, Osterhout G, Dick J. Grouping of some clinically relevant gram-positive rods by automated fatty acid analysis. APMIS 1991; 99: 147-54.

18. Cummings LA, Wu WK, Larson AM, Gavin SE, Fine JS, Coyle MB Effects of media, atmosphere, and incubation time on colonial morphology of Arcanobacterium haemolyticum. J Clin Microbiol 1993; 31: 3223-6.

19. Carlson P, Lounatmaa K, Kontiainen S. Biotypes of Arcanobacterium haemolyticum. J Clin Microbiol 1994; 32: 1654-7.

20. Miller RA, Brancato F, Holmes KK. Corynebacterium haemolyticum as a cause of pharyngitis and scarlatiniform rash in young adults. Ann Intern Med 1986; 105: 867-72.

21. Clarridge JE. The recognition and significance of Arcanobacterium haemolyticum. Clin Microbiol Newsl 1989; 11: 41-5.
22. Washington JA, Martin WJ, Spiekerman RE. Brain abscess with Corynebacterium haemolyticum: report of a case. Am J Clin Pathol 1971; 56: $212-5$

23. Dethy M, Hantso P, Van-Bosterhaut B, Swine C, Sassine A. Septicemia caused by Arcanobacterium haemolyticum (Corynebacterium haemolyticum) and Streptococcus milleri. Acta Clin Belg 1986; 41: 115-8.

24. Dobinsky S, Noesselt T, Rücker A, Maerker J, Mack D. Three cases of Arcanobacterium haemolyticum associated with abscess formation and cellulitis. Eur J Microbiol Infect Dis 1999; 18: 804-6.

25. Givner LB, McGehee D, Taber LH, Stein F, Sumaya CV. Sinusitis, orbital cellulitis and polymicrobial bacteremia in a patient with primary Epstein-Barr virus infection. Pediatr Infect Dis J 1984; 3: 254-6.

26. Dieleman LA, de-Marie S, Mouton RP, Bloem JL, Peters WG, Bos AJ, et al. Paravertebral abscess due to nondiphteria coryneform bacteria as a complication of ingrow toenails. Infection 1989; 17: 26-7

27. Carlson P, Korpela M, Walder M, Nyman M. Antimicrobial susceptibilities and biotypes of Arcanobacterium haemolyticum blood isolates. Eur J Clin Microbiol Infect Dis 1999; 18: 915-7.

28. Chandrasekar PH, Molinari JA. Corynebacterium haemolyticum bacteremia with fatal neurologic complication in an intravenous drug addict. Am J Med 1987; 82: 638-40.

29. Worthington MG, Daly BDT, Smith FE. Corynebacterium haemolyticum endocarditis on a native valve. South Med J 1985; 78: 1261-2.

30. Jobanputra RS, Swain CP. Septicaemia due to Arcanobacterium haemolyticum. J Clin Pathol 1975; 28: 798-800.

31. Waller KS, Johnson J, Wood BP. Radiological case of the month. Cavitary pneumonia due to Arcanobacterium haemolyticum. Am J Dis Child 1991; 145: 209-10.

32. Hoosen AA, Rasool MN, Roux L. Posttraumatic ankle joint infection with Arcanobacterium haemolyticum: a case report. J Infect Dis 1990; 162: $780-1$.

33. Krech T, Hollis DG. Corynebacterium and related organisms. En: Balows A, Hauser HL, Herrmann KL Jr, Isemberg ID, Shadomy HJ, eds. Manual of clinical microbiology, 5th ed. Washington: American Society for Microbiology, 1991; 277-86.

34. Brander MA, Jousimies-Somer HR. Evaluation of the RapID ANA II and API ZYM systems for identification of Actinomyces specimens. J Clin Microbiol 1992; 30: 3112-6.

35. Gavin SE, Leonard RB, Briselden AM, Coyle MB. Evaluation of the rapid CORYNE identification system for Corynebacterium species and others coryneforms. J Clin Microbiol 1992; 30: 1692-5.

36. Freney J, Duperron MT, Courtier C, Hansen W, Allard F, Boeufgras $\mathrm{JM}$, et al. Evaluation of API Coryne in comparision with conventional methods for identifying coryneform bacteria. J Clin Microbiol 1992; 29 38-41.

37. Kämpfer P. Differentiation of Corynebacterium spp., and related organisms by using fluorogenics substrates. J Clin Microbiol 1992; 30: 1067-71.

38. Nyman M, Banck G, Thore M. Penicillin tolerance in Arcanobacterium haemolyticum. J Infect Dis 1990; 161: 261-5

39. Kovatch AL, Schuit KE, Michaels RH. Corynebacterium haemolyticum peritonsillar abscess mimicking diphteria. JAMA 1983; 249: 1757-8.

40. Österlund A. Are penicillin treatment failures in Arcanobacterium haemolyticum pharyngotonsillitis caused by intracellular residing bacteria? Scand J Infect Dis 1995; 27: 131-4. 